\title{
Design of Honeycomb Microperforated Structure with Adjustable Sound Absorption Performance
}

\author{
Shanlin Yan $\mathbb{D}^{1},{ }^{1}$ Jinwu Wu $\mathbb{D}^{1},{ }^{1}$ Jie Chen $\mathbb{D}^{,},{ }^{1}$ Qibo Mao, ${ }^{1}$ and Xiang Zhang $\mathbb{D}^{2}$ \\ ${ }^{1}$ School of Aircraft Engineering, Nanchang Hangkong University, Nanchang 330063, China \\ ${ }^{2}$ School of Mechanical and Electrical Engineering, Harbin Institute of Technology, Harbin 150006, China \\ Correspondence should be addressed to Jinwu Wu; wujinwu@nchu.edu.cn
}

Received 19 December 2020; Revised 31 May 2021; Accepted 11 June 2021; Published 22 June 2021

Academic Editor: Selda Oterkus

Copyright (c) 2021 Shanlin Yan et al. This is an open access article distributed under the Creative Commons Attribution License, which permits unrestricted use, distribution, and reproduction in any medium, provided the original work is properly cited.

In this work, a double-layer honeycomb microperforated structure with adjustable back-cavity's height is designed based on cylinder honeycomb structure and microperforated panel (MPP). The sound absorption performance can be changed by adjusting the height of back-cavity. Thus, a better absorption performance is achieved by changing the position of the inner MPP. Acoustic impedance of the structure was calculated based on transfer matrix method. The sound absorption coefficient of the structure was obtained by finite element method (FEM). Meanwhile, the 3D printing technology was used to produce the experimental samples. The experimental results demonstrate that the sound absorption coefficient of the structure is greater than 0.8 in the range of $750-1250 \mathrm{~Hz}$, greater than 0.9 in the range of $2297-3592 \mathrm{~Hz}$, and above 0.5 in the range of $500-4000 \mathrm{~Hz}$. In addition, the feasibility of achieving adjustable sound absorption by means of changing the height of the back-cavity is verified by theoretical, simulation, and experimental results. The structure proposed in this work can realize the function of wide-band and better sound absorption performance by changing the position of the inner MPP, which can be applied to effectively reduce different frequencies noise.

\section{Introduction}

The ubiquitous noise problem has become a significant issue in today's society. From daily living environment to military equipment, structures and equipment used for noise reduction can be seen everywhere, such as noise reduction headphones $[1,2]$, sound insulation layer of room walls $[3,4]$, noise reduction in aircraft combustion chambers [5], and acoustic liner [6, 7]. Honeycomb microperforated panel (HMPP) structure is one of the noise reduction structures [8]. It combines simple structures and significant noise reduction performance of MPP with high specific strength and good fatigue resistance. Meanwhile, it forms a lightweight but high-strength composite structure which can reduce noise in harsh environments $[9,10]$. However, the sound absorption performance of this noise reducer is limited by its own structure, materials, and other factors.

In recent years, in order to improve its noise reduction performance, scholars have made improvements in different aspects. Kim et al. used the analysis method, revealed the influence of each layer of perforated panels on the sound absorption performance, and came up with an improvement measure [11]. Gai et al. designed an L-shaped back-cavity to enhance the sound-absorbing performance of the structure [12]. Zhang et al. designed a corrugated honeycomb microperforated composite structure by changing the internal propagation path of sound waves, which could effectively improve the noise reduction performance [13]. Xie et al. designed a honeycomb microperforated structure with different apertures, which refined the sound absorption performance [14]. By means of parallel mechanical impedance, Zhao improved the sound absorption performance of MPP in low frequency [15]. Scholars have done a lot of research on acoustic superstructure, metamaterials and made great progress, such as phonon crystal, chiral material, and local resonance acoustic metamaterial [16-19]. Xiong used optimization algorithm to design a multiarea honeycomb microperforated structure, which greatly improves the sound absorption bandwidth of the structure. However, the optimized parameters cannot be accurately processed and 
manufactured [20]. Although the sound absorption performance of HMPP will be better after optimizing the parameters, the sound absorption performance of the structure is determined completely. Even more, due to the difficulties in manufacturing, it could not be easily applied on a large scale.

In the process of increasing the noise reduction performance of the structure, scholars found that if the sound absorption performance of the structure was designed to be adjustable, the noise reduction capability of the structure would be improved. Shao designed an adjustable muffler based on the membrane structure [21]. Zhang et al. designed a honeycomb microperforated structure which can adjust the structure's sound absorption performance with the appropriate shape of inner panel. By changing the perforation rate, the purpose of adjustable sound absorption performance was achieved [22]. Ning et al. designed an adjustable resonance unit to control the width of the resonant band gap [23].

To sum up, there are many ways to improve the acoustic absorption performance of the MPP structure, but most research results are difficult to popularize in practical applications, such as too complex structures, production and manufacturing difficulties, and expensive defects. Therefore, in order to broaden the sound absorption frequency band of the sound absorber, under the premise that the size of the structure does not change, a double-layer HMPP with adjustable back-cavity height is designed and fabricated, which can efficiently improve the sound absorption performance and broaden the application range.

In the remainder of the paper, geometric model of the structure is designed and theoretical results are calculated by using two methods in Section 2. Next, Section 3 calculates the simulation results of the structure. Moreover, experimental results of samples are measured in Section 4 and compared with theoretical and simulation results. Finally, Section 5 concludes this work.

\section{Structural Acoustic Impedance}

Although the single-layer structure with microperforated sound absorber and honeycomb core has excellent sound absorption performance, its sound absorption frequency band is slightly narrower than the double-layer perforated panel. Figure 1 shows a single-layer HMPP structure. The structure is composed of MPP on the upper surface, a honeycomb core in the middle, and a bottom panel below [24].

The improved cellular structure of the honeycomb core is proposed to obtain better and adjustable sound absorption performance in this work. Its detailed structure is shown in Figure 2.

Under purpose of no change for the structure size, the screw and inner MPP are set in the unit cell of single-layer honeycomb structure which is shown in Figures 2(a) and 2(b). The inner thread of the MPP in the honeycomb core is matched with the screw and shown in Figure 2(c). The sectional view of the improved honeycomb core structure is shown in Figure 2(d). To retain the freedom in the vertical

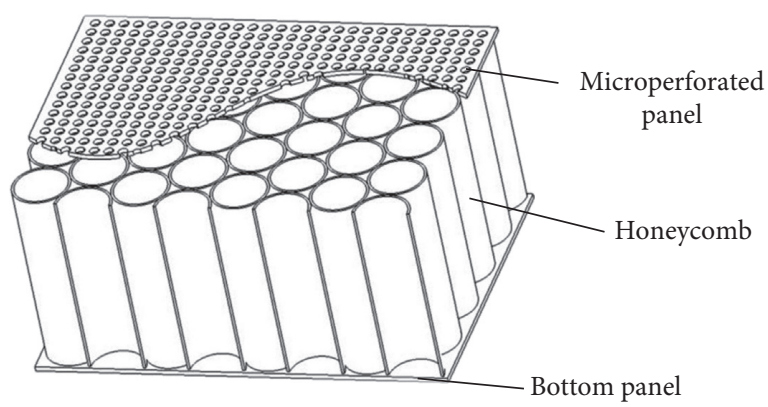

Figure 1: Single-layer HMPP.

direction, the convex strip on the inner wall of the honeycomb core, shown in Figure 2(e), and the grooves of the inner MPP, shown in Figure 2(f), coordinate each other to limit the freedom of the inner MPP. The inner MPP is equipped with $1 \mathrm{~mm}$ thickness and $5 \mathrm{~mm}$ height tubular structure to increase the contact area between the groove and honeycomb core, so as to better limit freedom within the rest of the direction of MPP. The diameter of the screw and bottom panel contact position is slightly smaller than that of the screw so that the screw is guaranteed to rotate freely while the inner microperforated panel moves up and down. As shown in Figure 2(g), the knob is connected with the screw inside the honeycomb core. Meanwhile, there is a pointer pointing to the scale values on the structure, which is convenient to control the rising height of the inner MPP. The outer wall of the knob has four grooves for better stress during rotation.

The inner MPP's position can change a screw pitch when the knob is rotated one circle. To be specific, the inner MPP rises by $1 \mathrm{~mm}$ when the knob is rotated a circle clockwise. Rotating the knob can change the position of the inner MPP, which further changes the height of the back-cavity of the structure and ultimately changes the frequency corresponding to the maximum sound absorption coefficient of the structure. So, the cavity heights $D_{1}$ and $D_{2}$ described in this work have a wide range of variation.

2.1. Acoustoelectric Analogy. According to Ma's theory of double-layer MPP and the method of acoustoelectric analogy, the equivalent sound source is the open circuit sound pressure and internal impedance which are $2 \mathrm{p}$ and $\rho_{0} c_{0}$. The acoustic impedance inside the honeycomb core can be regarded as the acoustic impedance of a doublelayer MPP [25]. The double-layer MPP and its equivalent circuit diagram are shown in Figure 3.where $r_{i}$ is the relative resistance of $M P P_{i}, m_{i}$ is the relative mass of $\mathrm{MPP}_{\mathrm{i}}$, $j$ is the imaginary unit, $\omega$ is the circular frequency of the incident noise, $Z_{D i}$ is the relative impedance of cavity, $D_{i}$ is the height of cavity, $\rho_{0}$ is the density of air, $c$ is the sound velocity in air at room temperature, $f$ is the frequency of sound, $\mu$ is the kinetic viscosity coefficient for air, $t_{i}$ is the panel thickness, $d_{i}$ is the perforation diameter, $p_{i}$ is the perforation rate, and $k_{i}$ is the characteristic coefficient of $\mathrm{MPP}_{i}$. 


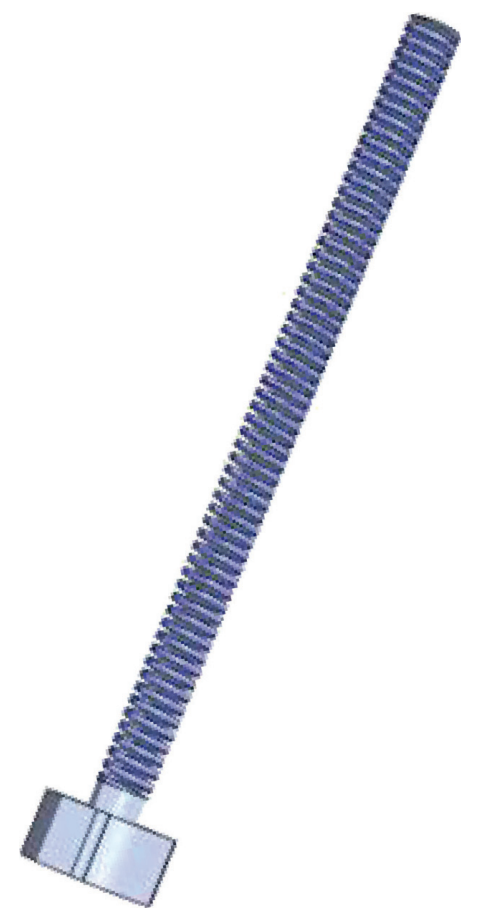

(a)

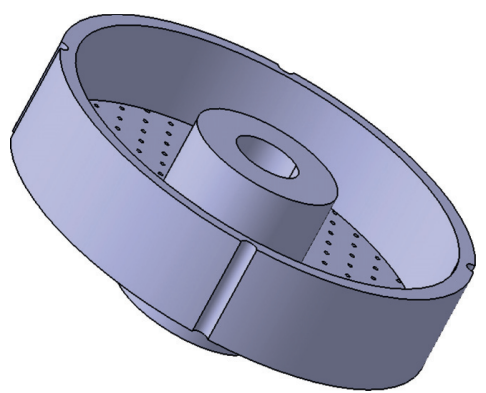

(b)

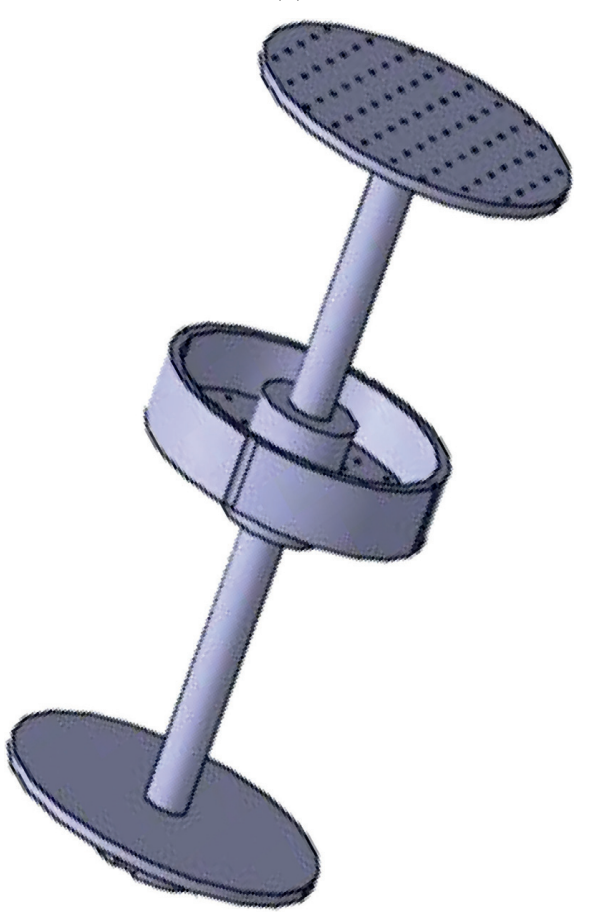

(c)

Figure 2: Continued. 


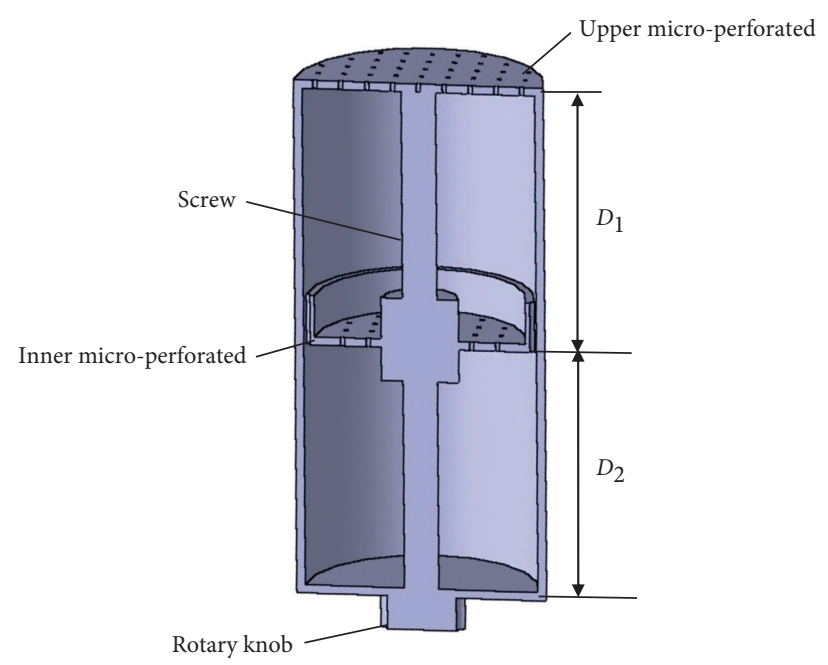

(d)

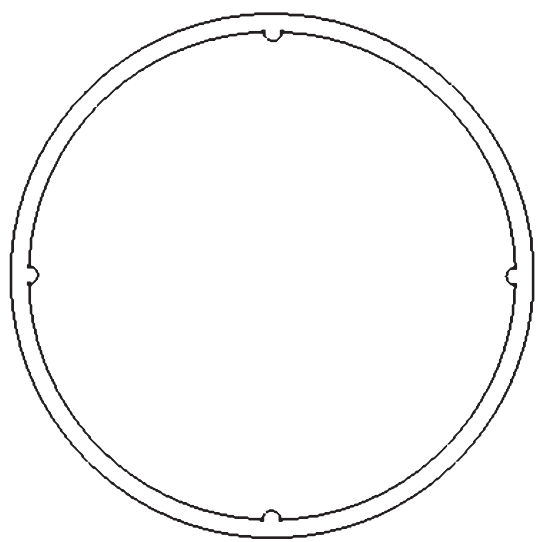

(e)

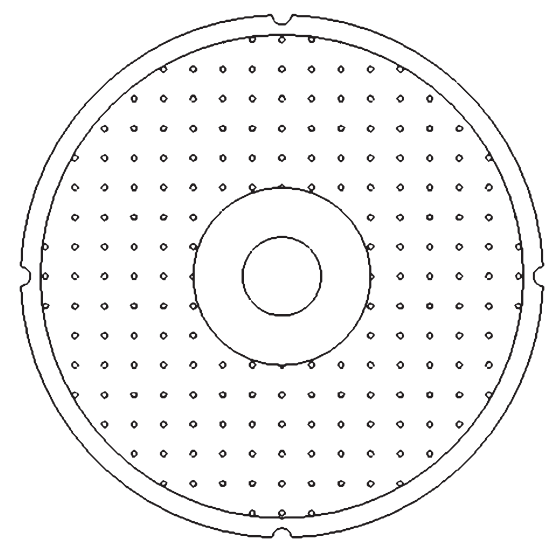

(f)

Figure 2: Continued. 


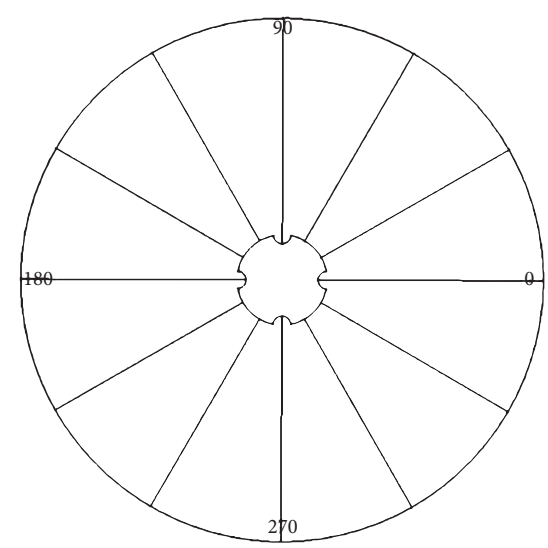

(g)

FigURE 2: Improved honeycomb core: (a) screw; (b) inner MPP; (c) honeycomb core internal structure; (d) honeycomb core section view; (e) top view of the honeycomb core; (f) top view of the inner MPP; (g) knob.
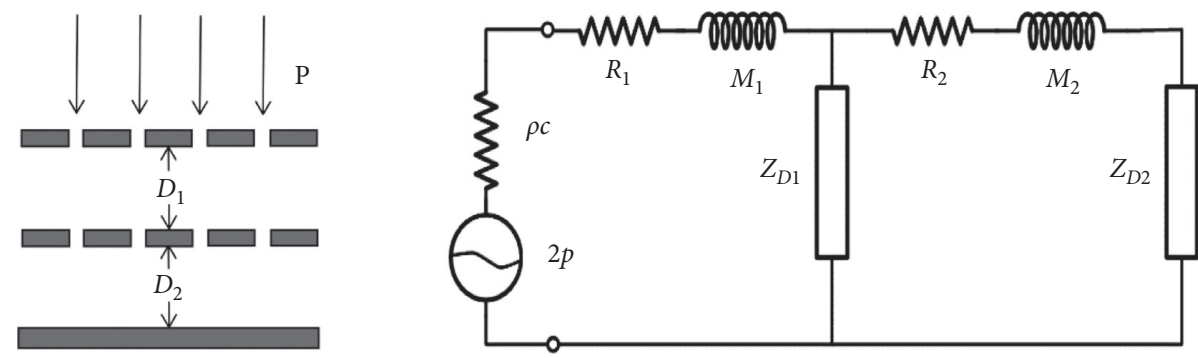

FIGURE 3: Double-layer MPP and its equivalent circuit diagram.

$$
\begin{aligned}
r_{i} & =\frac{32 \mu t_{i}}{\rho_{0} p_{i} d^{2}} \cdot\left\{\left(1+\frac{k^{2}}{32}\right)^{1 / 2}+\left(\frac{\sqrt{2}}{8} \frac{k d_{i}}{t_{i}}\right)\right\}, \\
m_{i} & =\frac{t_{i}}{p_{i} c} \cdot\left\{1+\left(9+\frac{k^{2}}{2}\right)^{-1 / 2}+\left(0.85 \frac{d_{i}}{t_{i}}\right)\right\}, \\
k_{i} & =\frac{d_{i}}{2} \sqrt{\frac{\omega}{\mu}} \\
Z_{D i} & =-j \cot \left(\frac{w D_{i}}{c}\right)
\end{aligned}
$$

So, the relative impedance of the structure can be written

$$
Z=r_{1}+j\left(\omega m_{1}-\cot \left(\frac{\omega D_{1}}{c}\right)\right)+\left(\frac{\cot ^{2}\left(\omega D_{1} / c\right)}{r_{2}+j\left(\omega m_{2}-\cot \left(\omega D_{1} / c\right)-\cot \left(\omega D_{2} / c\right)\right)}\right)
$$

where $\mathrm{MPP}_{1}$ is the upper MPP, $\mathrm{MPP}_{2}$ is the inner MPP, $D_{i}$ is the height of cavity, $D_{1}$ is the length between $\mathrm{MPP}_{1}$ and $\mathrm{MPP}_{2}$, and $D_{2}$ is the length between $\mathrm{MPP}_{2}$ and the bottom panel.
2.2. Transfer Matrix. Another method which can achieve calculation requirements is the transfer matrix method [25]. The matrix used in this work can be written as follows: 


$$
\begin{aligned}
T_{\mathrm{MPPi}} & =\left[\begin{array}{cc}
1 & Z_{\mathrm{MPPi}} \\
0 & 1
\end{array}\right], \\
Z_{\mathrm{MPPi}} & =\rho_{0} c\left(r_{i}+j \omega m_{i}\right), \\
k & =\frac{\omega}{c}, \\
T_{D i} & =\left[\begin{array}{cc}
\cos (k D i) & j \rho_{0} c \sin (k D i) \\
j \sin \left(\frac{k D i}{\rho_{0} c}\right) & \cos (k D i)
\end{array}\right],
\end{aligned}
$$

where $T_{\mathrm{MPPi}}$ is the transfer matrix of $\mathrm{MPP}_{\mathrm{i}}, Z_{\mathrm{MPPi}}$ is the impedance of $\mathrm{MPP}_{\mathrm{i}}, T_{D i}$ is the transfer matrix of the cavity, and $k$ is the wave number.

The total transfer matrix of the structure in this work can be written as

$$
T_{\text {total }}=\left[T_{\mathrm{MPP} 1}\right] \cdot\left[T_{D 1}\right] \cdot\left[T_{\mathrm{MPP} 1}\right] \cdot\left[T_{D 2}\right] .
$$

Then, the relative impedance can be written as follows:

$$
Z=\frac{T_{\text {total11 }}}{\rho_{0} c T_{\text {total } 21}} .
$$

Sound absorption coefficient $\alpha$ of normal incidence can be got by substituting equations (2) and (5) into the following equation:

$$
\alpha=\frac{4 \operatorname{Re}(Z)}{[1+\operatorname{Re}(Z)]^{2}+[\operatorname{Im}(Z)]^{2}} .
$$

Figure 4 shows the theoretical results of acoustoelectric analogy and transfer matrix model in MATLAB. The structure parameters used are shown in Table 1 . The heights of cavity $D_{1}$ and ${ }_{D}^{2}$ are $30 \mathrm{~mm}$.

As shown in Figure 4, when calculating the sound absorption coefficient of the double-layer MPP, the transfer matrix method is closer to the experiment results than the acoustoelectric analogy method; Zhang and Zhao had studied the differences between these two theoretical models. The neglect of the sound mass of the cavity leads to the inaccuracy of the acoustoelectric analogy method when considering above a certain frequency $[22,26]$. Therefore, in the following study of this work, the sound absorption coefficient of the structure will be calculated by transfer matrix method.

It can be seen from the above theoretical formulas and Figure 5 that the impedance of the structure is affected by the parameters of $\mathrm{MPP}_{\mathrm{i}}$ and the cavity heights $D_{1}$ and ${ }_{D}^{2}$. Assuming that the parameters of MPP are determined, the values of $D_{1}$ and ${ }_{D}^{2}$ directly affect the impedance value of the structure. The result in Figure 5 indeed confirms that the sound absorption performance of the structure can be adjusted by changing the values of $D_{1}$ and ${ }_{D}^{2}$.

\section{Finite Element Simulation}

Finite element method (FEM) is an effective method to study complex structures. The pressure acoustic (frequency domain) module of COMSOL Multiphysics 5.4 software is used for simulation analysis in this work. The parameters of MPP and screw parameters are, respectively, shown in Tables 1 and 2. The parameters of the heights $D_{1}$ and $D_{2}$ are shown in Table 3.

The model is divided into free tetrahedral meshes by using the meshing function in the software. The unit cell contains 50556 domain elements, 6840 boundary elements, and 700 edge elements [27]. The simulation grid is shown in Figures 6(a) and 6(b).

In simulation, position adjustment of the inner MPP leads to the height change of $D_{1}$ and $D_{2}$, finally achieving the purpose of changing the sound absorption performance. Figure 7 is the simulation result of sound absorption coefficient.

According to the simulation results of Figure 7, there are two peaks of sound absorption coefficient curves between $0 \mathrm{~Hz}$ and $4000 \mathrm{~Hz}$ in simulation. The first peaks of curves appear near $1000 \mathrm{~Hz}$, while the second ones move greatly with the changing of the position of inner MPP. The frequency corresponding to the peak value of the second absorption coefficient moves from $2000 \mathrm{~Hz}$ to $3000 \mathrm{~Hz}$ when $\mathrm{D}_{2}$ changes from $15 \mathrm{~mm}$ to $45 \mathrm{~mm}$, In addition, it shows a much better improvement of sound absorption coefficient with the value greater than 0.8 in the range of $600 \mathrm{~Hz}-$ $1100 \mathrm{~Hz}$, greater than 0.9 in the range of $2250-3250 \mathrm{~Hz}$, and greater than 0.6 in the range of $700-3500 \mathrm{~Hz}$.

\section{Experimental Verification}

In this work, the double-layer HMPP with adjustable height of back-cavity is fabricated by $3 \mathrm{D}$ printing technology. As shown in Figure 8, the honeycomb core structure with a diameter of $29 \mathrm{~mm}$ is selected for the sample which can be measured in an impedance tube according to ISO 10534-2 [28]. The detail parameters of samples are shown in Tables 1 and 2.

The sound absorption coefficient of the sample is obtained by the method of exchanging channels which means measuring the sound pressure at two fixed positions and the transfer function of the two channels. The total sound pressure at microphone 1 and microphone 2 is calculated as follows:

$$
\begin{aligned}
& p_{1}=p_{I} e^{j k(x+d)}+p_{R} e^{-j k(x+d)}, \\
& p_{2}=p_{I} e^{j k d}+p_{R} e^{-j k d},
\end{aligned}
$$

where $p_{I}$ is the amplitude of incident wave sound pressure, $d$ is the distance between microphone 2 and the front surface of the specimen, $p_{R}$ is the amplitude of reflected wave sound pressure, and $x$ is the distance between two microphones.

The transfer functions of incident $H_{1}$ and reflected waves $H_{R}$ can be expressed as. 


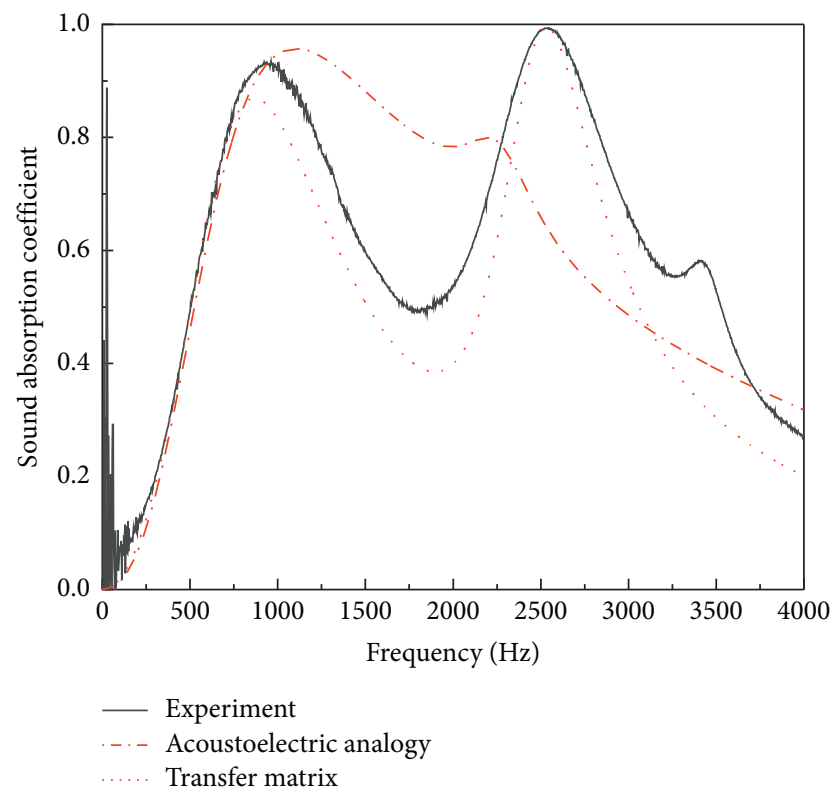

FiguRE 4: Experiment and acoustoelectric analogy and transfer matrix calculated results.

TABLE 1: Parameters of microperforated panels.

\begin{tabular}{lccc}
\hline Type & Board thickness $(\mathrm{mm})$ & Perforation ratio (\%) & Hole spacing $(\mathrm{mm})$ \\
\hline Upper MPP $\left(\mathrm{MPP}_{1}\right)$ & 1 & 4 & 0.35 \\
Inner MPP $\left(\mathrm{MPP}_{2}\right)$ & 1 & 8 & 0.35 \\
\hline
\end{tabular}

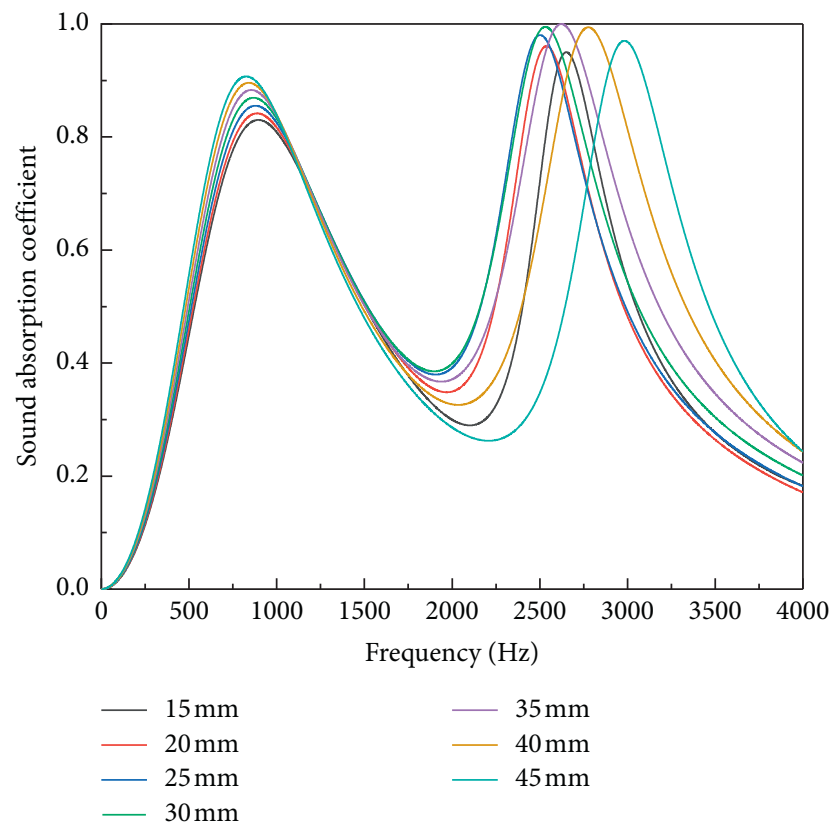

Figure 5: Transfer matrix calculated results.

TABLE 2: Screw parameters.

\begin{tabular}{lccc}
\hline Height $(\mathrm{mm})$ & External diameter $(\mathrm{mm})$ & Active length $(\mathrm{mm})$ & Screw pitch $(\mathrm{mm})$ \\
\hline 65 & 4 & 60 & 1
\end{tabular}


Table 3: Parameters of the back cavities.

\begin{tabular}{lcr}
\hline Example & $D_{1}$ & $D_{2}$ \\
\hline Example 1 & $15 \mathrm{~mm}$ & $45 \mathrm{~mm}$ \\
Example 2 & $20 \mathrm{~mm}$ & $40 \mathrm{~mm}$ \\
Example 3 & $25 \mathrm{~mm}$ & $35 \mathrm{~mm}$ \\
Example 4 & $30 \mathrm{~mm}$ & $30 \mathrm{~mm}$ \\
Example 5 & $35 \mathrm{~mm}$ & $25 \mathrm{~mm}$ \\
Example 6 & $40 \mathrm{~mm}$ & $20 \mathrm{~mm}$ \\
Example 7 & $45 \mathrm{~mm}$ & $15 \mathrm{~mm}$ \\
\hline
\end{tabular}

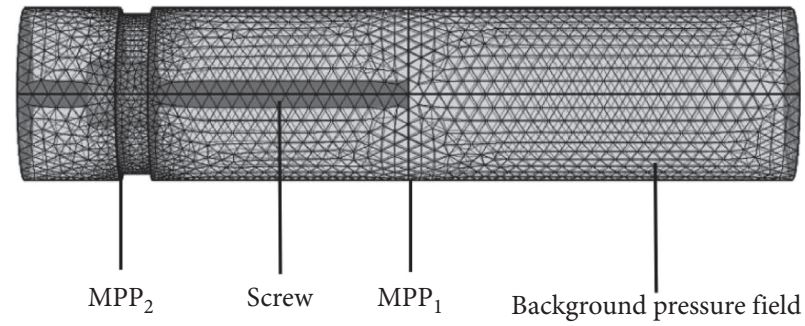

(a)

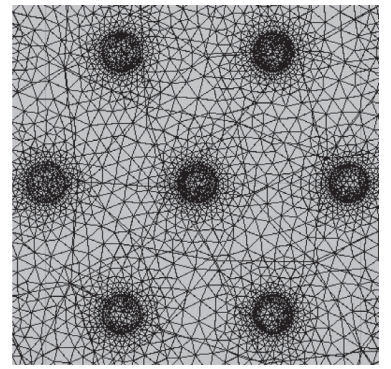

(b)

Figure 6: Finite element meshing: (a) finite element grid of $29 \mathrm{~mm}$ sample; (b) schematic diagram of micropore finite element grid.

$$
\begin{aligned}
H_{1} & =\frac{p_{21}}{p_{11}} \\
& =e^{-j k s}, \\
H_{R} & =\frac{p_{2 R}}{p_{1 R}} \\
& =e^{j k s} .
\end{aligned}
$$

The transfer function of the total sound field $H_{12}$ can be obtained from $p_{1}$ and $p_{2}$. Thus, the sound absorption coefficient $\alpha$ can be written as.

$$
\begin{aligned}
\alpha & =1-|r|^{2} \\
& =1-\left|\frac{H_{12}-H_{1}}{H_{R}-H_{12}} e^{j 2 k(x+d)}\right|^{2} .
\end{aligned}
$$

The AWA6290T transfer function acoustic absorption coefficient measurement system of Hangzhou Aihua Instrument Co., Ltd., is adopted in this work. The test schematic diagram and measurement system are shown in Figure 9. The sensitivity of the sensor needs to be calibrated before the experiment begins so that the sound pressure amplitude at frequency of $1000 \mathrm{~Hz}$ is about $94 \mathrm{db}$. In this test, the adjusted sensor sensitivity level is -48.55 .

Figure 10 shows the experimental sound absorption performance of the sample when $D_{2}$ changes from $15 \mathrm{~mm}$ to $45 \mathrm{~mm}$. The test frequencies range from 0 to $4000 \mathrm{~Hz}$ for samples. It can be found in Figure 10 that the sound absorption coefficient changed with the changing of position of inner MPP. Furthermore, there are two peaks of sound absorption coefficient between $500 \mathrm{~Hz}$ and $4000 \mathrm{~Hz}$ for

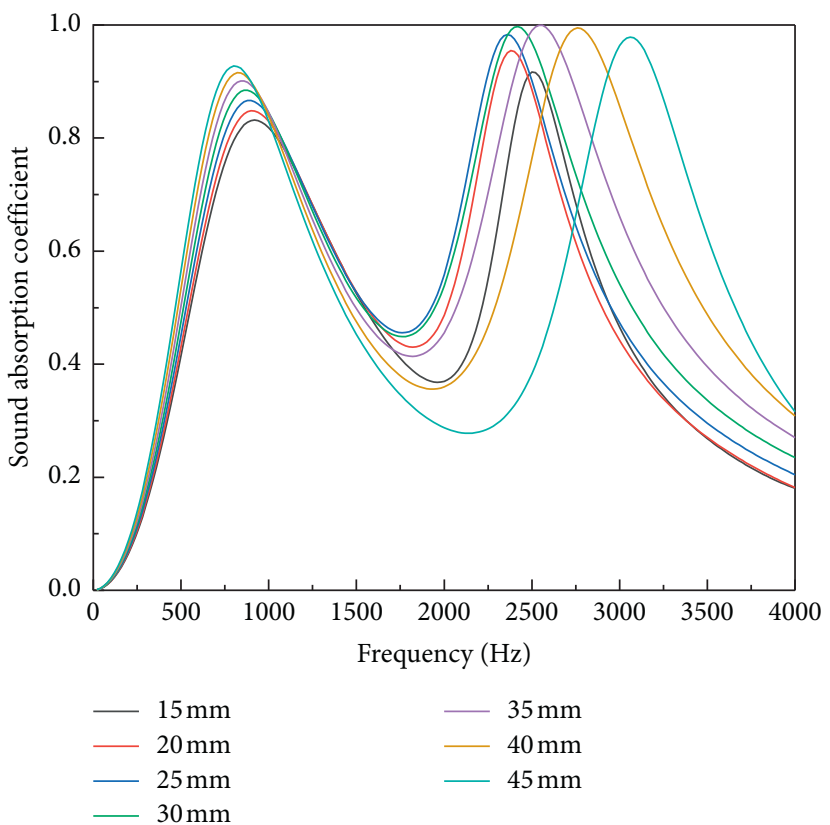

Figure 7: Simulation result of sound absorption coefficient.

samples, and when $D_{2}$ changes from $15 \mathrm{~mm}$ to $45 \mathrm{~mm}$, the sound absorption coefficient of the structure in the range of $2297-3592 \mathrm{~Hz}$ is above 0.9 with the width of $1295 \mathrm{~Hz}$ and is above 0.5 in the range of $500-4000 \mathrm{~Hz}$.

The frequencies corresponding to the first peak and the second peak of the sound absorption coefficient are extracted from the results. Considering the inner MPP at different heights from the bottom surface, the results can be drawn as Figure 11. 


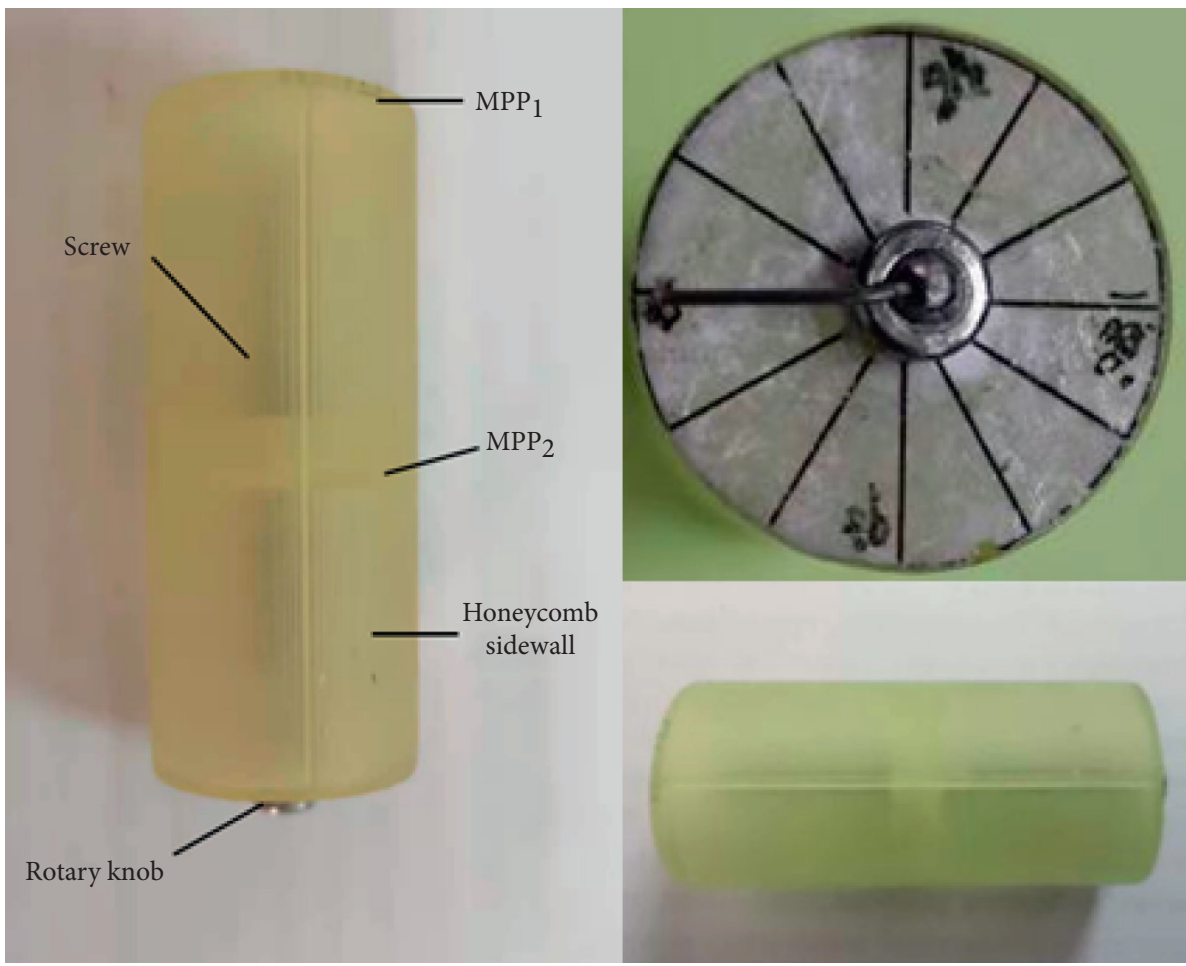

FIGURE 8: 3D printing samples.

As shown in Figure 11, the first peak frequency of the sound absorption coefficient moves to the low frequencies when the height of the cavity 1 becomes smaller and the height of the cavity 2 increases; however, the frequency corresponding to the peak of the second sound absorption coefficient moves to the high frequency. It proves that the sound absorption performance of this structure can be controlled by changing the height of the back-cavity.

It is obviously seem in Figure 12 that theoretical and simulation results have the same changes as the experimental results. Meanwhile, in the theoretical and simulation results, the first maximum sound absorption coefficient corresponds to the same frequency, but the maximum sound absorption coefficient is slightly lower than the experimental results. For the frequency corresponding to the second maximum absorption coefficient, theoretical and experiment results have the same frequency, while the simulation results move slightly to the low frequency, and the maximum sound absorption coefficient is the same in the three methods.

As shown in Figure 13, the frequencies corresponding to the first peak and the second peak in theoretical and simulation results of samples were respectively compared with the experimental results. There are errors in the $3 \mathrm{D}$ printed MPP, so the actual size of holes on MPP is slightly larger than the predetermined size, which made the resonant frequencies of the structure move towards high frequencies. But the results of the three methods show that the structure can be used to adjust the sound absorption performance, so as to indirectly expand the sound absorption band.

\section{Discussion and Conclusions}

The main factors affecting the sound absorption performance of MPP are the cavity depth, aperture, thickness, and perforation rate of MPP. If the other three parameters are held constant, the resonant frequency of the structure is changed by changing the cavity depth, so as to achieve the purpose of adjustable sound absorption performance.

(1) According to the theory of double-layer MPP, the change of the peak frequency of sound absorption coefficient depends on the change of the back-cavity's height of the structure, which shows the feasibility of adjustable sound absorption performance of the structure

(2) The height of the inner MPP can be controlled and adjusted by turning the knob to achieve the purpose of adjusting the sound absorption performance. The sound absorption coefficient of the structure in the range of $750-1250 \mathrm{~Hz}$ is above $0.8,2297-3592 \mathrm{~Hz}$ is above 0.9 , and $500-4000 \mathrm{~Hz}$ is above 0.5 .

(3) Theoretical, simulation, and experimental results have the same change rules. As the diameters of the microhole produced by $3 \mathrm{D}$ printing in the experiment are bigger than those of the theoretical and simulation results, the peak frequency in the experiment moves to the high frequency. Nonetheless, the sound absorption performance of the structure can still be predicted through theoretical or simulation results. 


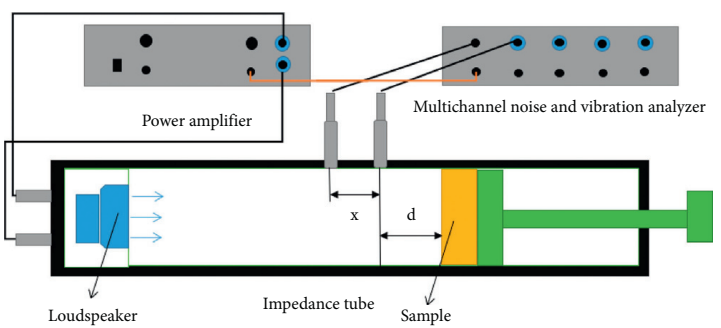

(a)

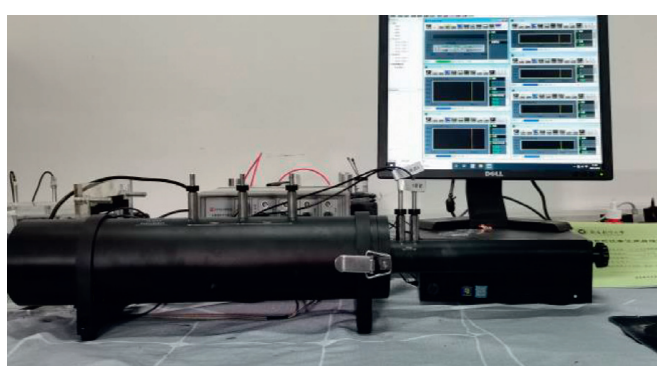

(b)

FIgURE 9: Schematic diagram of experimental measurement: (a) test schematic diagram of transfer function method; (b) AWA6290T transfer function acoustic absorption coefficient measurement system.

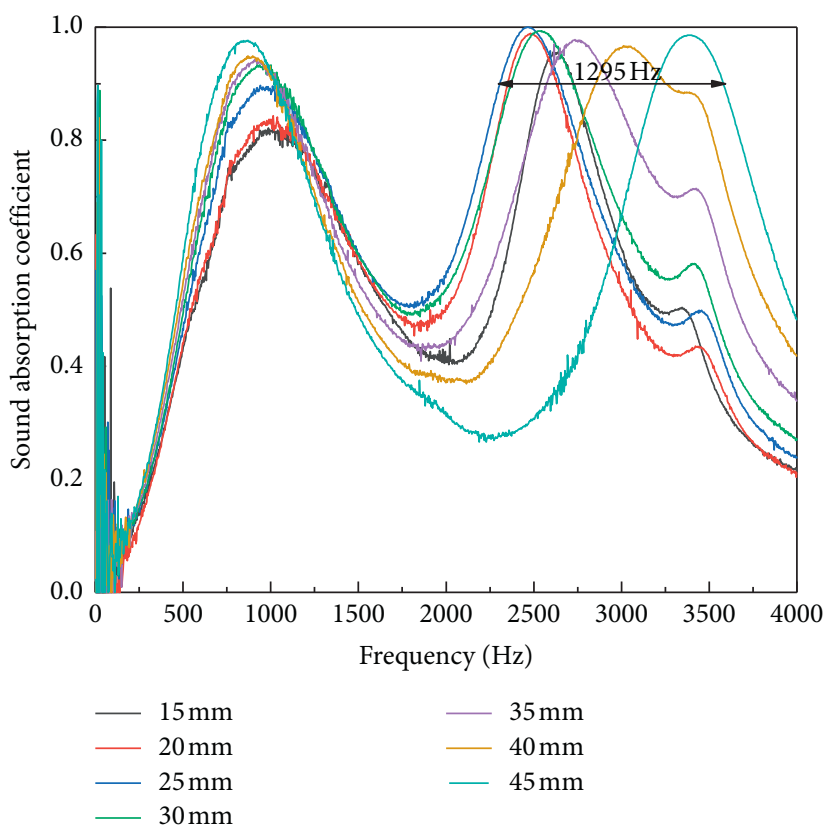

Figure 10: Experimental results.

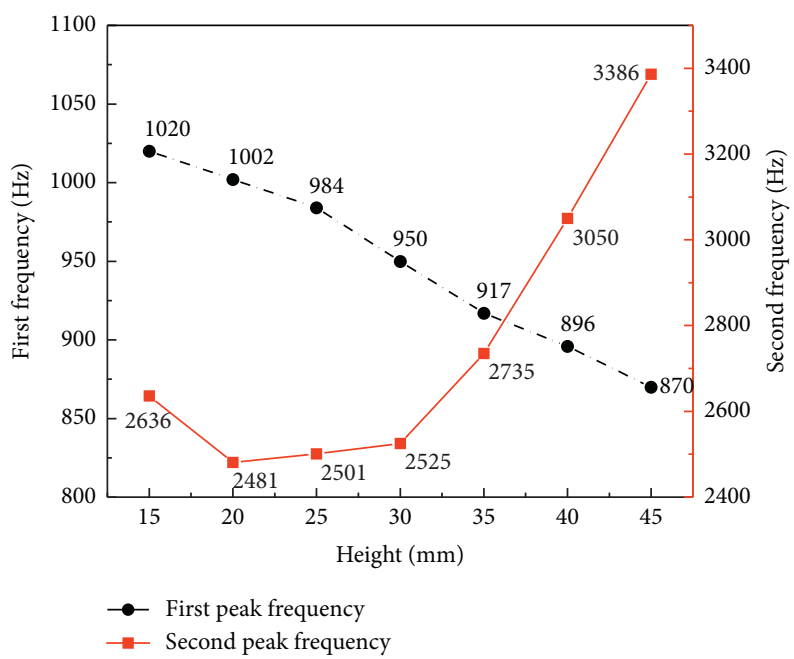

FIGURE 11: The relationship between the peak frequency and inner microperforated panel's position. 


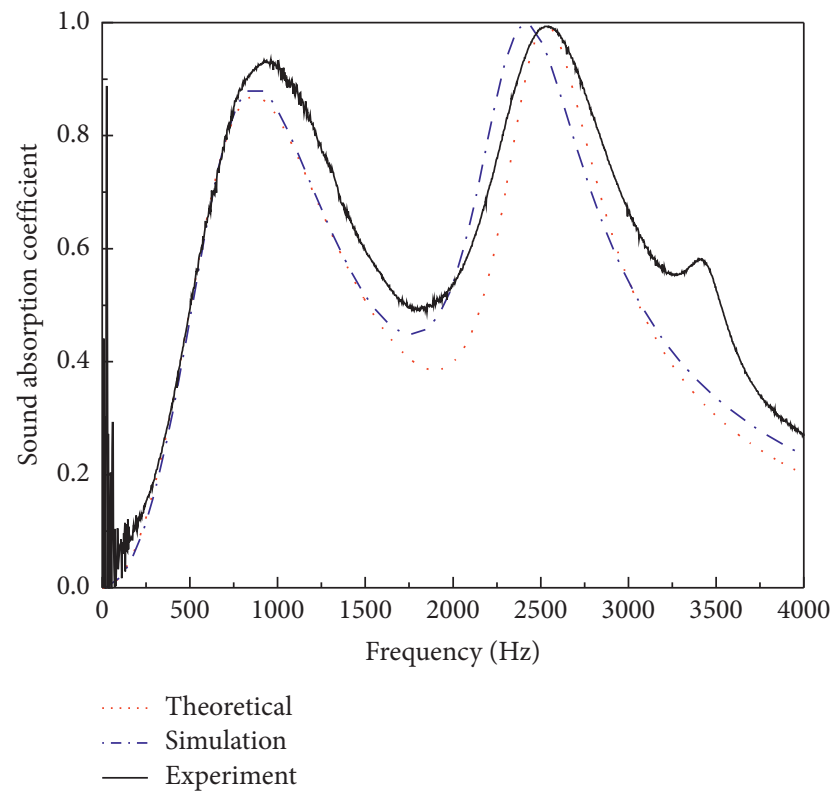

FIGURE 12: Results of three methods were compared when the inner MPP was $30 \mathrm{~mm}$ from the bottom.

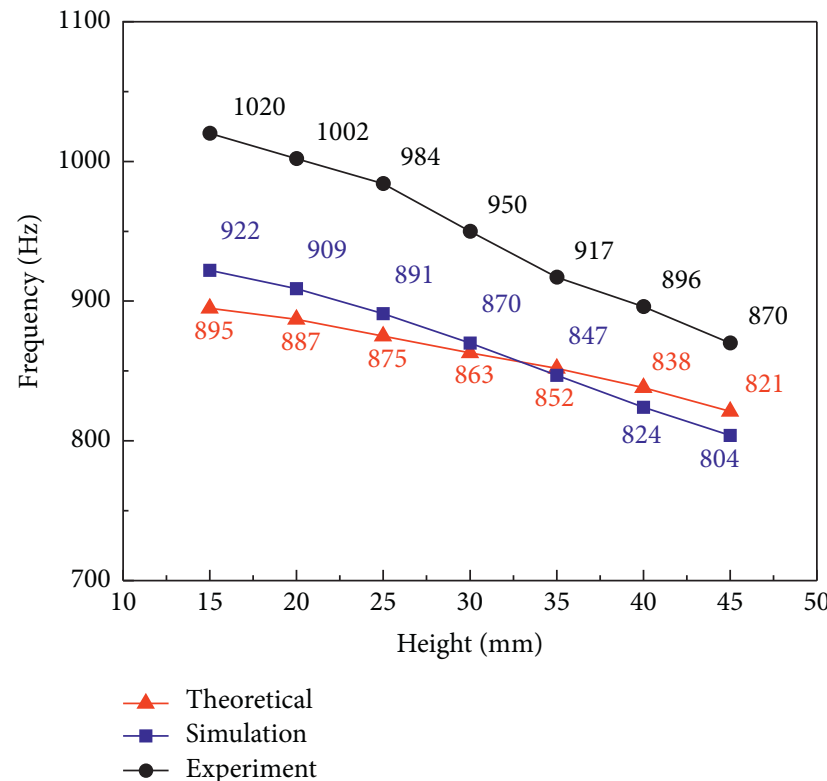

(a)

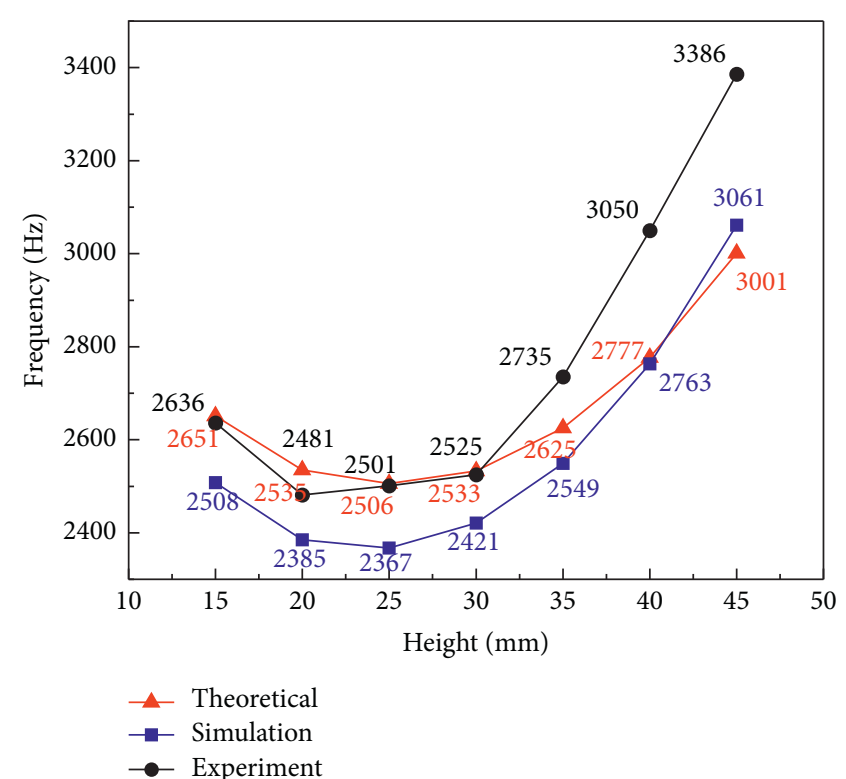

(b)

FIgURE 13: Comparison diagram of peak frequency of the three methods: (a) first peak frequency; (b) second peak frequency.

(4) The sound absorption performance of HMPP can be controlled and adjusted by adding a screw which controls the position of the inner MPP. When the sound absorber structures' parameters are limited, compared with the traditional sound absorption structure, the double-layer HMPP with adjustable sound absorption performance can be more widely applied.

\section{Data Availability}

Relevant research data can be obtained upon request from the corresponding author.

\section{Conflicts of Interest}

The authors declare that they have no conflicts of interest. 


\section{Acknowledgments}

This work was supported by the National Natural Science Foundation of China (51965041) and Innovation Special Fund Project of Nanchang Hangkong University (YC2020050).

\section{References}

[1] L. Y. L. Ang, Y. K. Koh, and H. P. Lee, “The performance of active noise-canceling headphones in different noise environments," Applied Acoustics, vol. 122, pp. 16-22, 2017.

[2] M. Liang, F. Zhao, D. French, and Y. Zheng, "Characteristics of noise-canceling headphones to reduce the hearing hazard for MP3 users," Journal of the Acoustical Society of America, vol. 131, no. 6, pp. 4526-4534, 2012.

[3] M. Ascigil-Dincer and S. Yilmaz Demirkale, "Sound insulation laboratory measurements of hollow brick masonry walls with different cavity depths and filling thickness," Noise Control Engineering Journal, vol. 63, no. 3, pp. 225-238, 2015.

[4] C. Calleri, A. Astolfi, L. Shtrepi et al., "Characterization of the sound insulation properties of a two-layers lightweight concrete innovative façade," Applied Acoustics, vol. 145, pp. 267-277, 2019.

[5] D. Zhao and X. Y. Li, "A review of acoustic dampers applied to combustion chambers in aerospace industry," Progress in Aerospace Sciences, vol. 74, pp. 114-130, 2015.

[6] B. S. Beck, N. H. Schiller, and M. G. Jones, "Impedance assessment of a dual-resonance acoustic liner," Applied Acoustics, vol. 93, pp. 15-22, 2015.

[7] Q. Li and D. Yang, "Vibration and sound transmission performance of sandwich panels with uniform and gradient auxetic double arrowhead honeycomb cores," Shock and Vibration, vol. 2019, Article ID 6795271, 16 pages, 2019.

[8] K. Hoshi, T. Hanyu, T. Okuzono et al., "Implementation experiment of a honeycomb-backed MPP sound absorber in a meeting room," Applied Acoustics, vol. 157, Article ID 107000, 2020.

[9] M. Thota and K. W. Wang, "Tunable waveguiding in origami phononic structures," Journal of Sound and Vibration, vol. 430, pp. 93-100, 2018.

[10] D. Zhao, E. Gutmark, and A. Reinecke, "Mitigating self-excited flame pulsating and thermoacoustic oscillations using perforated liners," Science Bulletin, vol. 64, no. 13, pp. 941-952, 2019.

[11] H.-S. Kim, P.-S. Ma, S.-R. Kim, S.-H. Lee, and Y.-H. Seo, “A model for the sound absorption coefficient of multi-layered elastic micro-perforated plates," Journal of Sound and Vibration, vol. 430, pp. 75-92, 2018.

[12] X.-L. Gai, T. Xing, X.-H. Li et al., "Sound absorption of microperforated panel with 1 shape division cavity structure," Applied Acoustics, vol. 122, pp. 41-50, 2017.

[13] F. H. Zhang, Y. F. Tang, F. X. Xin, and T. J. Lu, "Microperforated acoustic metamaterial with honeycomb-corrugation hybrid core for broadband low frequency sound absorption," Acta Physica Sinica, vol. 67, no. 23, Article ID 234302, 2018

[14] S. Xie, D. Wang, Z. Feng, and S. Yang, "Sound absorption performance of microperforated honeycomb metasurface panels with a combination of multiple orifice diameters," Applied Acoustics, vol. 158, Article ID 107046, 2020.

[15] X.-D. Zhao, X. Wang, and Y.-J. Yu, "Enhancing low-frequency sound absorption of micro-perforated panel absorbers by combining parallel mechanical impedance," Applied Acoustics, vol. 130, pp. 300-304, 2018.

[16] I. Barba, A. Grande, G. J. Molina-Cuberos, Á. J. GarcíaCollado, J. Represa, and A. C. L. Cabeceira, "A full-dielectric chiral material based on a honeycomb structure," International Journal of Antennas and Propagation, vol. 2018, Article ID 4198243, 6 pages, 2018.

[17] P. Gulia and A. Gupta, "Sound attenuation in triple panel using locally resonant sonic crystal and porous material," Applied Acoustics, vol. 156, pp. 113-119, 2019.

[18] V. Romero-García, A. Krynkin, L. M. Garcia-Raffi, O. Umnova, and J. V. Sánchez-Pérez, "Multi-resonant scatterers in sonic crystals: locally multi-resonant acoustic metamaterial," Journal of Sound and Vibration, vol. 332, no. 1, pp. 184-198, 2013.

[19] K. Shi, G. Jin, R. Liu, T. Ye, and Y. Xue, "Underwater sound absorption performance of acoustic metamaterials with multilayered locally resonant scatterers," Results in Physics, vol. 12, pp. 132-142, 2019.

[20] Y. Xiong, "Optimal design of broadband sound absorption performance of honeycomb microperforated sound absorber," Technical Acoustics, vol. 41, no. 3, 2022.

[21] H. Shao, H. He, Y. Chen, X. Tan, and G. Chen, "A tunable metamaterial muffler with a membrane structure based on Helmholtz cavities," Applied Acoustics, vol. 157, Article ID 107022, 2020.

[22] X. Zhang, J. W. Wu, Q. B. Mao, W. Zhou, and Y. Xiong, "Design of a honeycomb-microperforated panel with an adjustable sound absorption frequency," Applied Acoustics, vol. 164, Article ID 107246, 2020.

[23] S. Ning, F. Yang, C. Luo, Z. Liu, and Z. Zhuang, "Low-frequency tunable locally resonant band gaps in acoustic metamaterials through large deformation," Extreme Mechanics Letters, vol. 35, Article ID 100623, 2020.

[24] A. Omrani and I. Tawfiq, "Vibro-acoustic analysis of microperforated sandwich structure used in space craft industry," Mechanical Systems and Signal Processing, vol. 25, no. 2, pp. 657-666, 2011

[25] D. Y. Ma, "Wide-band sound absorber based on microperforated panels," Chinese Journal of Acoustics, vol. 05, pp. 197-208, 1985.

[26] X. D. Zhao and Y. Y. Zhao, "Improvement and experiment of transfer matrix calculation method for micro-perforated plate," Technical Acoustics, vol. 28, no. 2, pp. 164-167, 2009.

[27] D. Guan, D. Zhao, and Z. X. Ren, "Aeroacoustic attenuation performance of a helmholtz resonator with a rigid baffle implemented in the presence of a grazing flow," International Journal of Aerospace Engineering, vol. 2020, Article ID 1916239, 16 pages, 2020.

[28] ISO, Acoustics-determination of Sound Absorption Coefficient and Impedance in Impedance Tubes-Part 2: Transfer Function Method, Techstreet, Chicago, Illinois, 1998. 